\title{
Anomalous Left Main Coronary Artery: Not Always a Simple Surgical Reimplantation
}

\author{
Asif H. Khan · Ian B. A. Menown • Alastair Graham · John A. Purvis
}

To view enhanced content go to www.cardiologytherapy-open.com

Received: March 24, 2015 / Published online: May 5, 2015

(C) The Author(s) 2015. This article is published with open access at Springerlink.com

\section{ABSTRACT}

We present the case of 56-year-old woman who required complex coronary artery bypass grafting for high-risk anomalous left main coronary artery (LMCA) originating from right coronary cusp including conventional reimplantation of the LMCA plus left internal mammary artery (LIMA) graft to the left anterior descending (LAD) and saphenous vein graft (SVG) to the left circumflex (LCx). On subsequent cardiac computed tomography screening and cardiac catheterization, the LIMA graft was occluded after just a few centimeters, but the SVG graft was patent with

Electronic supplementary material The online version of this article (doi:10.1007/s40119-015-0039-x) contains supplementary material, which is available to authorized users.

A. H. Khan · I. B. A. Menown ( $\square)$

Craigavon Cardiac Centre, Southern Trust,

Craigavon BT63 5QQ, Northern Ireland, UK

e-mail: ian.menown@southerntrust.hscni.net

A. Graham

Royal Victoria Hospital, Belfast Trust, Belfast,

Northern Ireland, UK

J. A. Purvis

Altnagelvin Hospital, Western Trust, Londonderry,

Northern Ireland, UK good run-off into the native LCx and also filled the LAD retrogradely. The reimplanted left main stem demonstrated at least moderate ostial stenosis although pressure wire assessment of this was not significant (fractional flow reserve 0.89), probably due to good retrograde filling of the LAD from the SVG to LCx, therefore, we did not proceed with ostial LMCA stenting. She remains on yearly review with a low threshold for further revascularization should the SVG to LCx develop progressive stenosis. This case illustrates how patients with anomalous LMCA may sometimes benefit from grafting in addition to conventional reimplantation.

Keywords: Anomalous left main coronary artery; Cardiac catheterization; Cardiac computed tomography; Coronary artery bypass grafting; Pressure wire assessment; Right coronary cusp

\section{INTRODUCTION}

The majority of coronary artery anomalies (80\%) are benign and asymptomatic [1]. The incidence of an anomalous left main coronary 
artery (LMCA) originating from the right coronary cusp is low (0.05\%) [2] But if the artery takes an inter-arterial course between the aorta and the pulmonary artery, sudden death may take place by compression during vigorous exercise [3]. Standard management includes surgical reimplantation of the left main ostium. We report a case of LMCA with an anomalous origin from the right coronary cusp, surgical management strategy and invasive follow-up.

\section{CASE REPORT}

A 56-year-old Caucasian lady presented to our Rapid Access Chest Pain clinic following 3 episodes of non-specific sharp central chest discomfort over the previous 6 weeks. She had occasional palpations, but no history of presyncope or collapse.

On Bruce protocol exercise treadmill test, there was no ST segment depression, but frequent ventricular ectopics at peak exercise. Transthoracic echocardiography showed severe hypokinesis of basal, inferior and inferoapical segments with mild reduction in left ventricular ejection fraction (45\%). Given the ventricular ectopics and unexpected reduction in ejection fraction, cardiac catheterization was undertaken which showed no obstructive coronary artery disease (CAD) at rest, but the LMCA had an anomalous origin arising from right coronary cusp (Fig. 1a-b). Subsequent cardiac computed tomography (CT) angiography (Fig. 2) showed this anomalous course was a high-risk type, running between the aortic root and pulmonary artery although it was not compressed at rest and maintained a circular cross-section. The LMCA then ran anteriorly in an unusual course to reach the interventricular groove about onethird of the way down, at which point it trifurcated into mid and distal left anterior descending (LAD), a small ectatic diagonal
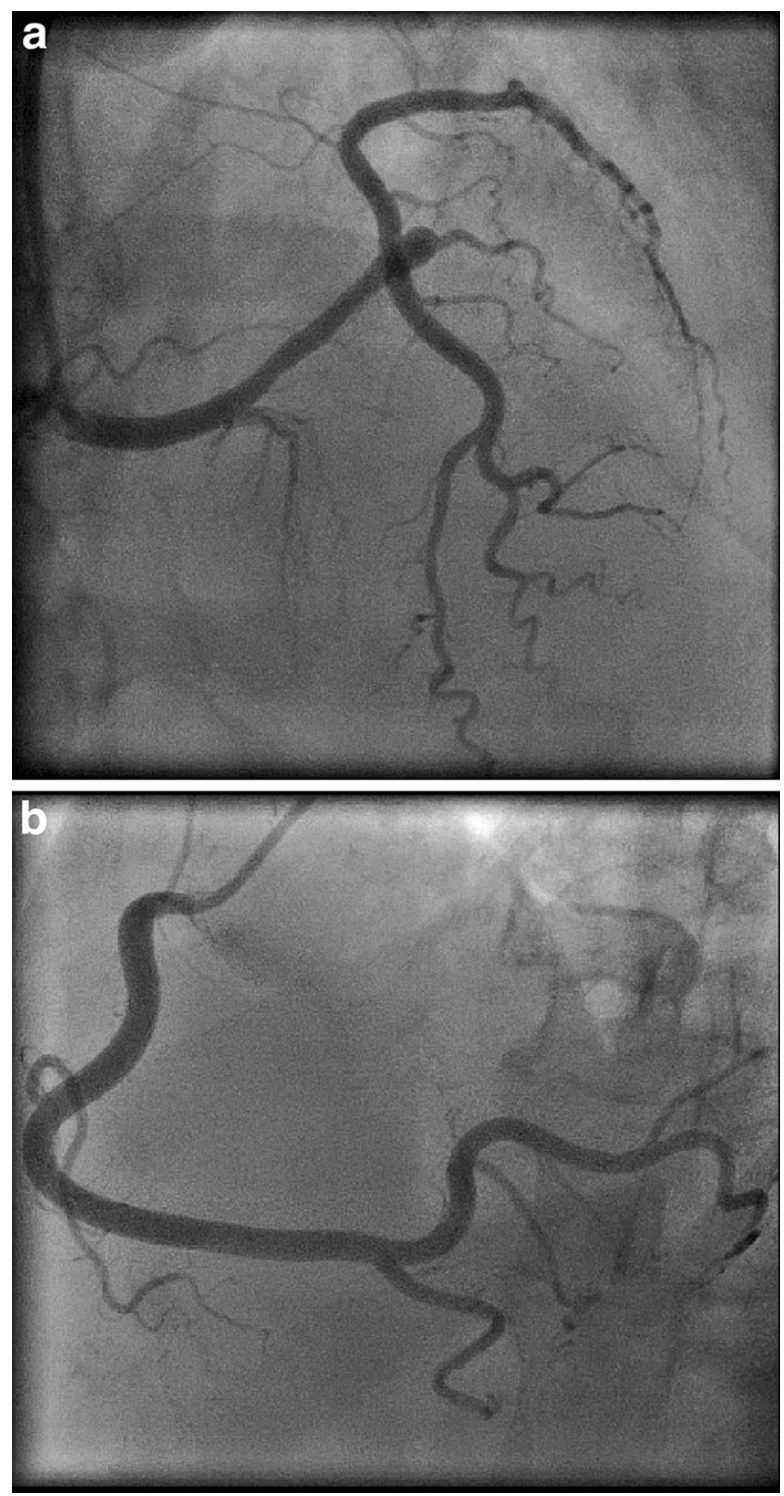

Fig. 1 Coronary angiogram at initial diagnosis: a anomalous left main coronary artery arising from the right coronary cusp and $\mathbf{b}$ normal right coronary artery

branch, and a proximal LAD that ran up the interventricular groove before turning to become the left circumflex (LCx). As this cardiac anatomy is considered high risk for life-threatening complications including sudden death, the patient was discussed by the heart team. The consensus was that surgery should be performed and that reimplantation of the anomalous LMCA was the best option. 


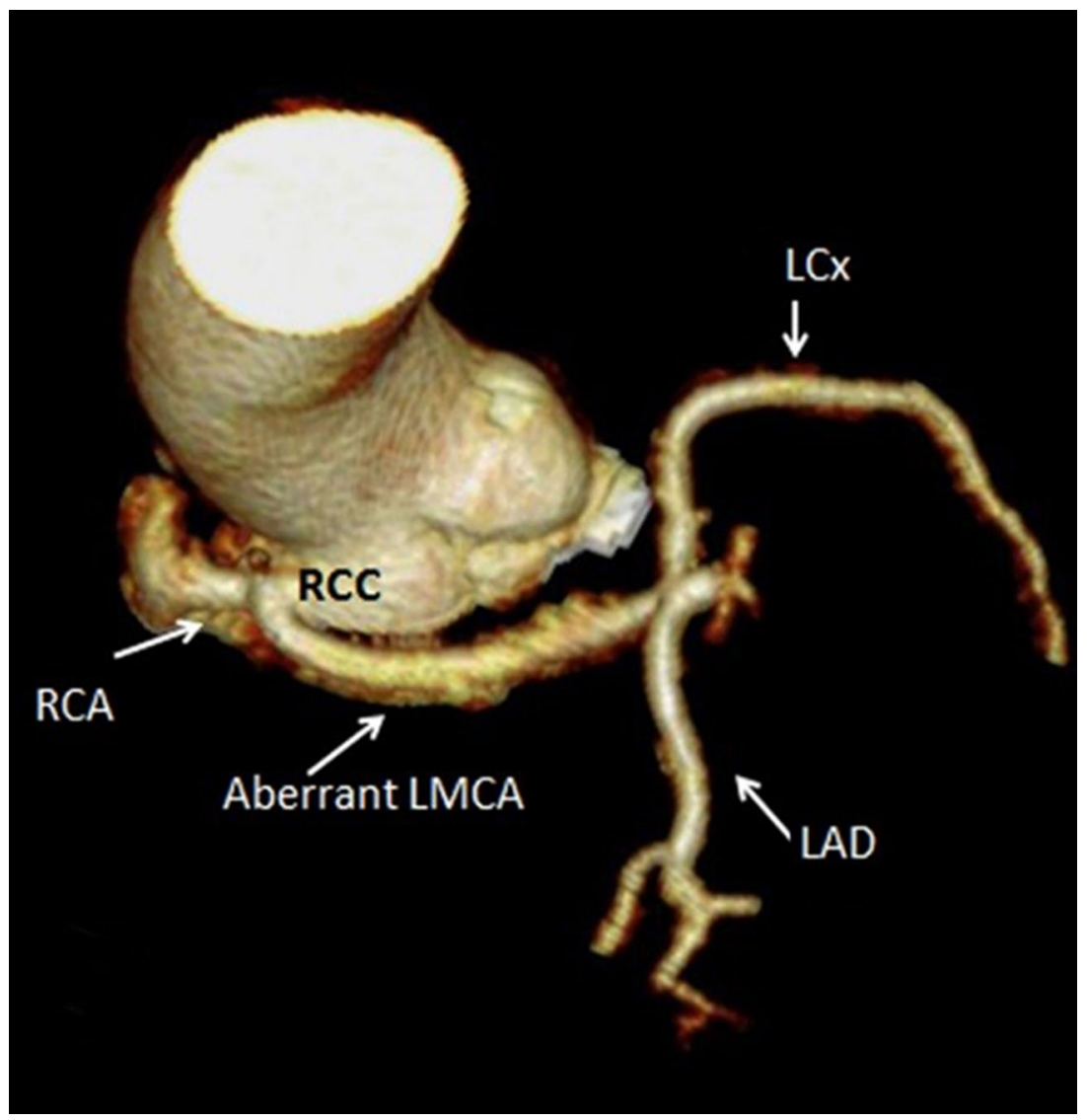

Fig. 2 Cardiac computed tomography pre-cardiac surgery showing the anomalous (aberrant) left main coronary artery (LMCA) arising from the right coronary cusp. $L A D$

Surgery was performed with the proximal orifice of the left main stem reimplanted by conventional technique in the left coronary sinus in the normal position. However, due to the surgeon's concerns that there remained the possibility for compression of the distal vessel, a left internal mammary artery (LIMA) was grafted to the LAD as a backup. Attempted closure of the chest was accompanied by ventricular fibrillation (VF) so the sternum was splinted open. The patient remained stable and on day 5, a saphenous vein graft (SVG) was performed to the first obtuse marginal then the sternum was closed. The patient made a good recovery and left ventricular ejection fraction remained unchanged. left anterior descending, $L C x$ left circumflex, $R C A$ right coronary artery, $R C C$ right coronary cusp

Given the difficult post-operative course, a follow-up cardiac CT was arranged. The LIMA graft appeared atretic after just a few centimeters (Fig. 3a), but the SVG graft was patent with good run-off into the native LCX with retrograde filling of the LAD (Fig. 3b). The surgically reimplanted LMCA had ostial stenosis (Fig. 3c).

CT findings were confirmed with invasive angiography (Figs. $4 \mathrm{a}-\mathrm{c}$ ). Pressure wire assessment of reimplanted LMCA ostium yielded no significant stenosis (fractional flow reserve 0.89), probably due to the good retrograde filling of the LAD from the SVG to LCx. She remains under yearly review with a low threshold for further revascularization 

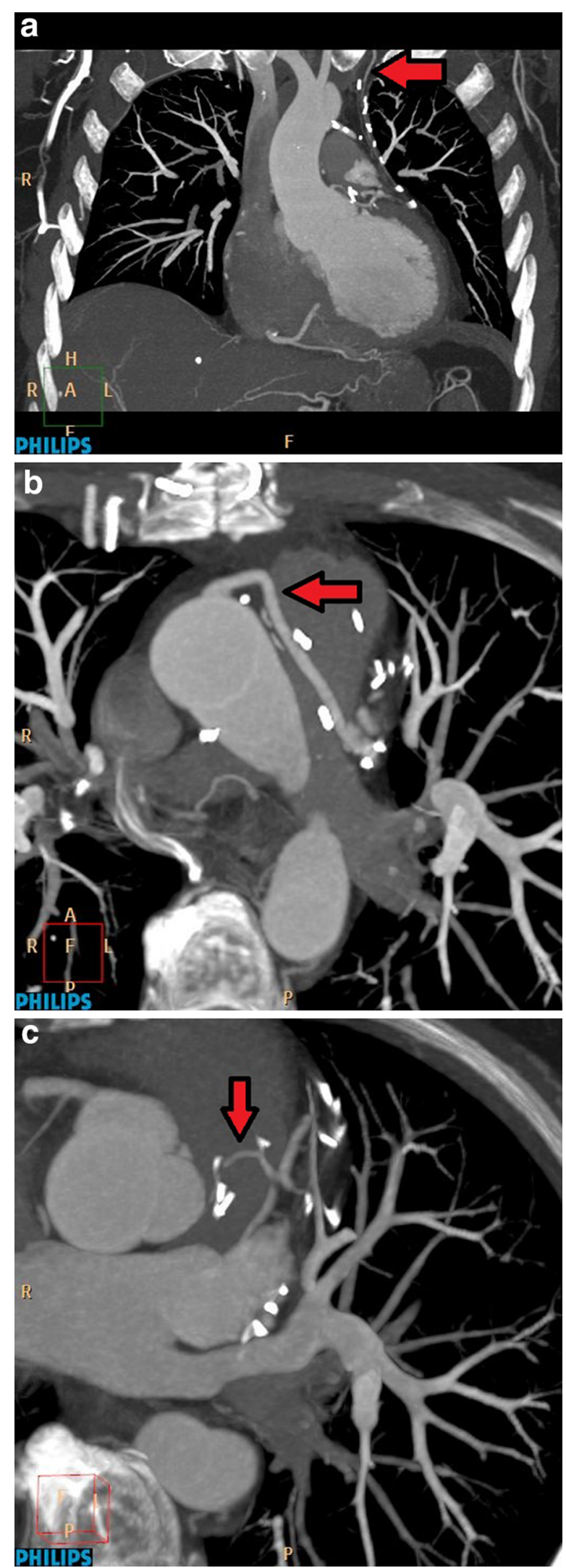

Fig. 3 Cardiac computed tomography imaging following cardiac surgery a left internal mammary artery, $\mathbf{b}$ vein graft to circumflex obtuse marginal branch and $\mathbf{c}$ reimplanted left main coronary artery

should the SVG to LCx develop progressive stenosis.

\section{DISCUSSION}

Anomalous LMCA with high-risk course is a rare, but important diagnosis. Accurate diagnosis can prevent the risk of sudden death and aid surgical planning. Most cases are unfortunately diagnosed post-mortem [4, 5].

This case demonstrates the difficulties in choosing the most appropriate surgical strategy. The initial operation was complicated by VF on closure of the chest despite the addition of an LIMA graft to the LAD. The surgeon remained concerned about the effect of competitive flow between reimplanted LMCA and the LIMA causing later occlusion of the graft. Potentially, the heart would be dependent on LIMA graft flow during occasions of LMCA compression such as vigorous exercise, but not at rest [6]. A vein graft was added to the first obtuse marginal branch of the LCx as it was the surgeon's belief that vein grafts are more resistant to the effects of competitive flow than internal mammary artery grafts due to a reduction in flow-related contractility although it is acknowledged that firm evidence for this is lacking.

Coronary angiography remains the gold standard for the evaluation of CAD. However, in the case of coronary anomalies, further evaluation by Cardiac CT or cardiac magnetic resonance imaging is recommended to determine the course of the anomaly and risk stratification [7]. 

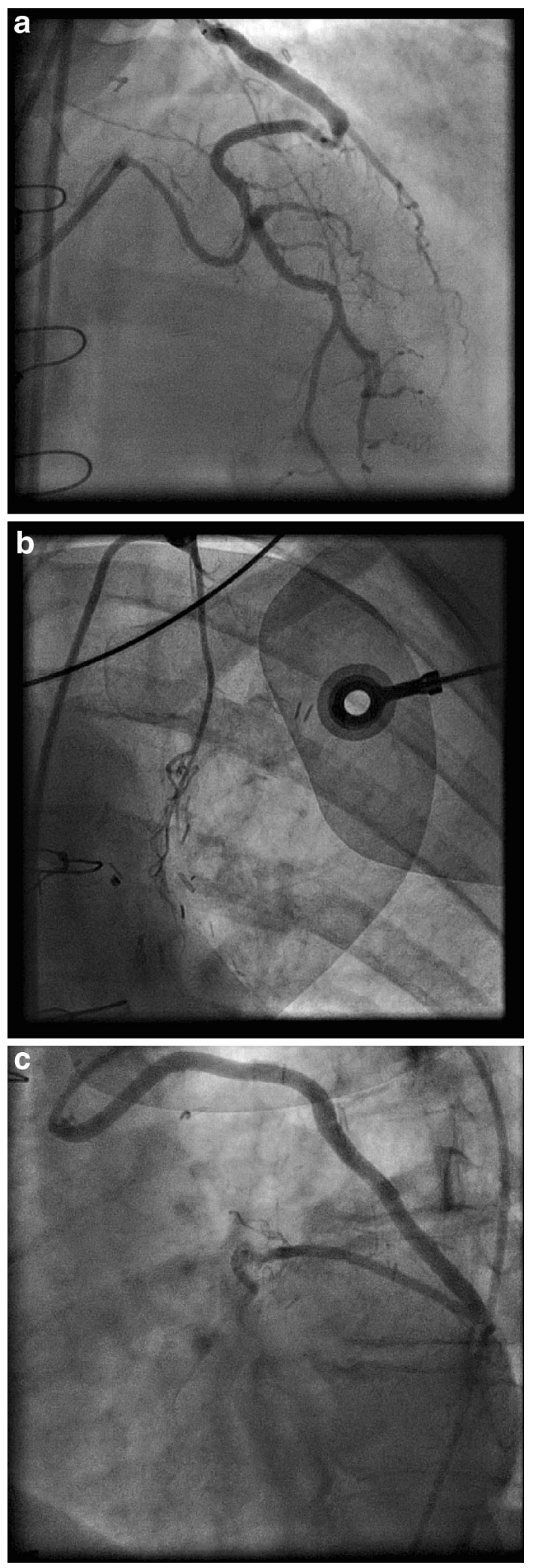

Fig. 4 Coronary angiogram following cardiac surgery a reimplanted left main coronary artery, b left internal mammary artery and $\mathbf{c}$ vein graft to circumflex obtuse marginal branch

Coronary artery bypass is considered as main surgical treatment option for reimplantation of high-risk anomalous coronary arteries [8]. Our case showed that a flexible surgical approach may be needed.

\section{CONCLUSION}

Anomalous origin coronary arteries should be worked up comprehensively and an aggressive treatment strategy adopted, especially if there is an inter-arterial course. Non-invasive and invasive modalities can assess the success of a complex surgical reimplantation strategy.

\section{ACKNOWLEDGMENTS}

No funding or sponsorship was received for publication of this article. All named authors meet the International Committee of Medical Journal Editors (ICMJE) criteria for authorship for this manuscript, take responsibility for the integrity of the work as a whole, and have given final approval for the version to be published.

Conflict of interest. Asif H. Khan, Ian B. A. Menown, Alastair Graham and John A. Purvis declare no conflicts of interest.

Compliance with ethics guidelines. Informed consent was obtained from the patient for publication of their data.

Open Access. This article is distributed under the terms of the Creative Commons Attribution Noncommercial License which 
permits any noncommercial use, distribution, and reproduction in any medium, provided the original author(s) and the source are credited.

\section{REFERENCES}

1. Yamanaka $\mathrm{O}$, Hobbs RE. Coronary artery anomalies in 126,595 patients undergoing coronary arteriography. Cathet Cardiovasc Diagn. 1990;21:28-40.

2. Desmet W, Vanhaecke J, Vrolix M, Van de Werf F, Piessens J, Willems $\mathrm{J}$, de Geest $\mathrm{H}$. Isolated single coronary artery: a review of 50,000 consecutive coronary angiographies. Eur Heart J. 1992;13:1637-40.

3. Basso C, Corrado D, Thiene G. Congenital coronary artery anomalies as an important cause of sudden death in the young. Cardiol Rev. 2001;9:312-7.

4. Frescura C, Basso C, Thiene G, Corrado D, Pennelli T, Angelini A, Daliento L. Anomalous origin of coronary arteries and risk of sudden death: a study based on an autopsy population of congenital heart disease. Hum Pathol. 1998;29(7):689-95.

5. De Rosa G, Piastra M, Pardeo M, Caresta E, Capelli A. Exercise-unrelated sudden death as the first event of anomalous origin of the left coronary artery from the right aortic sinus. J Emerg Med. 2005;29(4):437-41.

6. Kawamura M, Nakajima H, Kobayashi J, Funatsu T, Otsuka Y, Yagihara T, Kitamura S. Patency rate of the internal thoracic artery to the left anterior descending artery bypass is reduced by competitive flow from the concomitant saphenous vein graft in the left coronary artery. Eur J Cardiothorac Surg. 2008;34(4):833-8. doi:10.1016/j.ejcts.2008.07.011 (epub 2008 Aug 23).

7. Torres FS, Nguyen ET, Dennie CJ, Crean AM, Horlick E, Osten MD, Paul N. Role of MDCT coronary angiography in the evaluation of septal vs interarterial course of anomalous left coronary arteries. J Cardiovasc Comput Tomogr. 2010;4(4):246-54.

8. Thomas D, Salloum J, Montalescot G, Drobinski G, Artigou JY, Grosgogeat Y. Anomalous coronary arteries coursing between the aorta and pulmonary trunk: clinical indications for coronary artery bypass. Eur Heart J. 1991;12:832-4. 